\title{
A Review of Picture Based Psychological Assessment tools for children and adolescents
}

\author{
Snigdhasree Bhattacharya ${ }^{1}$, Uma Hirisave ${ }^{2}$ \\ ${ }^{1} \mathrm{PhD}$ Scholar, Department of Clinical Psychology, NIMHANS - INI, Bangalore. \\ ${ }^{2}$ Professor, Department of Clinical Psychology, NIMHANS - INI, Bangalore. \\ E-mail-snigdhab1@yahoo.com
}

\begin{abstract}
Children connect better with pictures than they do to verbal material. The review traces the application of pictures in the area of psychological assessment with children and adolescents. Pictures have been used as testing material in the domain of projective testing and self report formats. The pictorial tests available in each of the domains have been reviewed and separate analysis has been provided for each domain. The review focuses on the advantages of pictorial measures, their scope in the Indian context and challenges in development of pictorial measures.
\end{abstract}

Keywords: Pictorial Tools, Psychological Assessment using pictures, Assessment with children and adolescents.

\section{INTRODUCTION}

"If a picture paints a thousand words, then a let a picture inspire a thousand words." Nicholas Boyd Crutchley

Images and Drawings have been used to elicit responses in therapeutic consultations and in therapy. These tests are known as 'stimulus tests', evolved into combinations of stimuli and responses, creating a more interactive give and take between therapist and patient especially when working with children. A social constructivist approach views images as a visual resource that assists in the co-construction of understanding and meaning on a topic of interest, emotional and cognitive lives of the children [1]. It has been seen that children connect better to pictures than they do to words, a prime example of which is the use of illustrations in various books both academic and non academic. A drawing or photograph evokes a different kind of response and is favored for its immediacy and accessibility. The picture 'stands alone', as it were, in the immediacy of its presence. Images have a way of maintaining the present tense and so are powerful ways to represent events, people and meaning as if they were still happening [2]. This nature of the stimuli of drawings makes it a creative and valuable tool in psychological assessment. While paper and pencil tests are applicable, assessment with children and adolescents demand more innovative techniques which are both stimulating and interactive. Various creative techniques for assessment with children include storytelling, play, drama, social mapping, Story stem cards and use of drawings or pictures.

These methods draw on inventive and imaginative processes. They can serve as constructivist tools to assist research participants to describe and analyze their experiences and give meaning to them [3]. Eminent developmental psychologist Vygotsky posits that from about 7 years, children begin to master symbolic meaning of drawing and that drawing can serve as a cultural tool, in the 
same way as signs and language, for the mediation and transmission of an experience [4]. Pictures have been used in psychological assessment with children for over half a century now. Pictures are verbally less loaded and not threatening as it's in third person, yet relatable. Apart from this it is particularly useful with younger children, children who have been through any form of abuse, children who may either have difficulties or may not know how to read and write and children with problems like expressive speech delay, autism spectrum disorders or elective mutism. It is useful in these populations as the testing would have lesser emphasis on abilities of articulation or reading and would involve basic pointing.

Pictures have been integral in assessment of cognitive functions, projective testing as well as rating scales. The present article aims to review pictorial tools that are popularly used around the world and in India. The article was conceptualized as there is no compilation/ review of pictorial tools. As mentioned earlier, pictorial tools have immense utility in psychological assessment; therefore the current review will provide readers a detailed understanding about the various types and forms of pictorial tools available now and some that were previously available. The primary sources of the tests are based on articles, reviews and textbook descriptions of picture based psychological tools.

The beginnings of pictures in psychological assessment can be traced back to assessment of intellectual functioning. Use of pictures especially as a part of a subtest in assessment of intellectual functioning has been a common practice. However, very few tests solely rely on the use pictures in assessment of cognitive functions. One such test that completely relies on pictures for assessment of a student's receptive vocabulary is Peabody Picture Vocabulary Test (Currently PPVT-4) [5-8]. It was developed by Lloyd Dunn and his wife Leota Dunn and is currently in its $4^{\text {th }}$ edition and is co authored by their son Douglas M. Dunn. The test started off with black and white pictures and it currently uses colour pictures. It is an individually administered, untimed assessment where the student selects the picture that best represents an oral word. Apart from the regular English version Spanish, Braille, and large print versions are available. The time taken for administration is approximately 10-15 minutes. It is applicable for ages 2 years 6 month to 90years. The tool consists of 228 items equally distributed across 19 item-sets. Each item-set contains 12 items of increasing difficulty. The test has two forms, "Form A" and "Form B". The test has been standardized and found to have excellent psychometric properties.

An Indian adaptation of the PPVT was done by Verma in the year 1993.

The Picture Vocabulary Test (PVT) [9] was a part of the four test battery used to assess the cognitive development of children between ages of three and six years. The Gessels Drawing Test is also used as a part of the four test battery. The battery was constructed in India a part of an ICMR- WHO project on 'Indicators of mental health'. The tests can be used individually and not necessarily the complete battery. The picture vocabulary test comprises of 18 pictures which the child has to name. The score is the number of pictures correctly identified. Each score has a corresponding developmental age which is then used to calculate the developmental quotient. The test is very simple to administer and takes less than 10 minutes. The ease of administration had made it widely applicable in various research studies in India.

This clearly highlights, that the use of pictures in assessment of cognitive functions clearly eases administration as it is both less threatening and less time consuming. The tests measure a very practical output of cognitive functions i.e Visual Comprehension, giving the examinee a better chance at scoring better, thereby making the tests less biased. Since the tests use simple pictures of day to day life which are universal and not culture specific, it can be applied across various diverse cultures. These tests have better applicability in special populations like with children with developmental disorders, speech impairments, hearing impairments etc as it requires minimal output from the examinee. Therefore use of pictures in cognitive assessment has excellent applicability and scope.

\section{PICTURES IN PROJECTIVE TESTING}

Pictures have been an integral part of projective testing. However there has been a movement from abstract images like inkblots to concrete pictures depicting themes that are relevant to day to day. Use of pictures in psychological assessment was first used by Henry Murray in 1943 in the Thematic Apperception Test [10]. 
Thematic Apperception Test applies the 'projective hypothesis' which assumes that the way in which the examinee perceives and interprets the test material reflects fundamental aspects of his or her personality [11].

\section{THE THEMATIC APPERCEPTION TEST (TAT)}

This test was originally developed as an aide in therapy to reveal to dominant drives, emotions, sentiments, complexes and conflicts of a personality, a process that might otherwise take months of analysis. However over the years it has been useful in psychological assessment irrespective of the framework of reference of the therapist. The TAT contains 31 cards, including one blank card (card 16). The cards are designated as cards for males, boys, females, or girls. Although the TAT was developed in studies with adults, the manual states that the instrument may be used with children from 4 years of age and older. A survey showed that the TAT is frequently used with adolescents. The TAT manual provides some general guidelines for an evaluation of them responses. These guidelines are based on Murray's personality theory, which revolves around the concepts of "needs" (tendencies in the individual's personality) and "presses" (the individual's perception of forces from the environment). Over the years different scoring techniques have been developed and applied with TAT responses.

Like other projective techniques, the TAT has been criticized on the basis of poor psychometric properties. It must be noted that irrespective of criticisms a there are various reviewers who believe that traditional psychometric properties cannot be applied to a test like TAT. Despite criticisms, the TAT continues to be used as a tool for research, in clinical evaluation, as an aid in therapy and in others areas of applied psychology like organizational behavior and forensic psychology.

\section{INDIAN ADAPTATION OF THE TAT}

Indian Adaptation of TAT prepared by Dr Uma Chowdhary [12] according to the procedure laid down by McClelland by using TAT cards of Murray. The pictures have been modified for the Indian population and it consists of a set of 14 Cards. Of these, 12 are adapted from the original TAT and 2 additional ones representing themes important to Indian culture that has not been in the original TAT like joint family and religious fantasy. .The stimuli depict Indian characters wearing traditional Indian apparels but the situations are similar to the original cards. For Example - card 1 an Indian stringed instrument tanpura is substituted for a violin. Chowdhury compared the responses of 260 individuals on Rorschach results and TAT results and the responses were found to be congruent. The participants in the study hailed from different socio economic status and religions.

After, TAT, came a series of projective tests that used pictures. Thematic Apperception Tests became a genre in itself and has been reviewed in detail. [13] The tests that have used pictures and are projective in nature have been reviewed in this section.

\section{BLACKY PICTURES TEST}

The Blacky Pictures Test was created by Gerald Blum in 1947 [14]. The test consists of a series of twelve picture cards, to investigate the extent to which children's personalities were shaped by Freudian psychosexual development. The pictures show a family of cartoon dogs in normal situations. The main character, 'Blacky', was accompanied by a sibling Tippy, and by a mother and father. Blacky's gender was determined by the gender of the test subject. The stories narrated by the children were content analyzed for indications such as an anal personality, castration anxiety or penis envy. In 1950 several studies found results from the analysis of Blacky Pictures consistent with Freudian psychoanalytic theory, providing some support for the construct validity of the test. However, the test has been criticised for highly subjective scoring system. The test is currently out of print. 


\section{ROSENZWEIG PICTURE FRUSTRATION TEST FOR CHILDREN AND ADOLESCENTS}

The Rosenzweig Picture Frustration Test for Children and Adolescents [15] was developed by Saul Rosenzweig in the year 1948. The test consists of 24 cartoon pictures, each portraying two persons in a frustrating situation. Each picture contains two "speech balloons," a filled one for the "frustrator" or antagonist, and a blank one for the frustrated person, or protagonist. The subject is asked to fill in the blank balloon with his or her response to the situation, and the overall purpose of the study is to assess how the examinee responds to frustration and frustrating situations. The test is actually semi projective in nature as it involves an examinee responding verbally to a semi-ambiguous picture scenario and attempts to understand latent hostility through their responses. There are three forms available, child, adolescent and adult form. In terms of reliability and validity, the P-F Study inter rater reliability is in the range of 0.80 to 0.85 . The rest-retest reliability was found to be fair to marginal.

An Indian Adaptation of Rosenzweig Picture Frustration Test for Children [16] was developed by Udai Pareek in collaboration with Saul Rosenzweig in the year 1958. During the preparation of the Indian version, care was taken to retain the original situations. The 24 situations depicted in cartoon like pictures continue to be the same. However, changes made were mainly in form of certain paraphernalia of the pictures like clothing and furniture. Apart from this some of the captions were also changed and there was a slight modification in the record blank. The test has been standardized in 1000 children from 4 to 13 years of age. The test was found to have high inter rater reliability and moderate internal consistency. The test was further translated to seven Indian languages. However, it must be noted that the test has limited application in clinical settings due to restricted test findings.

\section{THE CHILDREN'S APPERCEPTION TEST (CAT)}

The Children's Apperception Test (Bellak \& Bellak, 1949/1991) [17] was developed for use with children aged 3 to 10 years. It consists of 10 black and white drawings showing anthropomorphized animals. A parallel version with human is available for older children. Apart from this a version for very young or retarded children depicts less common themes, such as parental separation, physical disability, and mother's pregnancy. Significant themes from psychodynamic developmental theory are clearly recognizable in the CAT cards (e.g., feeding problems, toilet training, oedipal feelings, sibling rivalry, and aggression). The administration of the test is standardized, has different scoring formats that have been established and has a strong theoretical grounding. While there are norms that have been established, the details are not well furnished.

Indian Adaptation of The Children's Apperception Test was also prepared by Dr Uma Chowdhary in 1985 [18]. Since CAT uses animal figures, it was initially expected to be culture free. However application of the original CAT in various parts of India, brought forward challenges that lesser privileged children experienced with the environmental details of the original CAT. Therefore efforts was made to keep it as close to the original CAT as possible with minor modifications, making it more relatable for Indian children. Eg - Replacing a pipe with hookah in Card 3, Indian style toilet instead of a western commode in Card 10. The test has commendable clinical usefulness as it provides the clinician an excellent insight about the child's conflicts.

\section{THE FOUR PICTURE TEST}

The Four Picture Test (van Lennep, 1948), [19] is a thematic apperception test from the Netherlands. The FPT is generally used in adult assessment, but the manual indicates that the test may be used with children aged 10 and older. The FPT has four postcard-sized plates that are presented together, and the examinee is asked to write a single story based on all four cards. The limitations of this test is that the images are not relatable by adolescents as they depict life in 1920's, lack of standardization and highly subjective scoring methods. 


\section{THE MICHIGAN PICTURE TEST}

The Michigan Picture Test (Andrew et al., 1953) [20] was developed jointly by psychologists at the University of Michigan and Michigan Department of mental Health in the late 1940's. The test consists of 15 cards and a blank card, 8 cards are common for both genders and fours cards are separate for boys and girls. It is applicable for children between 8 to 14 years of age. The test aims to understand the emotional adjustment of the child through story telling using the pictures as a lead. It has been used for both therapeutic as well as diagnostic purpose. The pictures are realistic and were found to be easily relatable by children in the process of standardization. The test has been well standardized on 1400 children throughout the state of Michigan. The test was found to have a high inter rater reliability (0.91 to 0.98$)$. However the test is not currently used and is out of print.

\section{THE PATTE NOIRE}

The Patte Noire (Corman, 1961) [13] is a thematic apperception test from France that is well known mostly in Europe. It is meant for children aged 5 years and up and uses animal figures. The postcard-sized cards show Patte Noire, a piglet with a black hind leg, in various situations, often together with other members of the pig family. A parallel version for Jewish and Arab populations depicts sheep instead of pigs. The theoretical framework of the Patte Noire focuses on Freudian psychosexual theory, and the cards clearly represent the stages of libidinal development in early childhood, namely, the passive oral stage (feeding, sibling rivalry, and abandonment), the active oral stage (aggression), the anal stage (cleanliness), and the Oedipal stage (parental relationships, fear of castration, and identification). The test has been popular, however it lacks empirical validation.

\section{THE COLOMBUS}

The Columbus (Langeveld, 1969) [21], named after the explorer is a test from the Netherlands. The test aims to understand the experiential world of the examinee. The Columbus was designed for children and adolescents from 6 to 18 years of age. The test consists of 21 black-and-white reproductions of watercolours and three coloured cards. The administration of the test is not standardized, lacks a clear scoring system and the European influence on the pictures makes it not very relatable by children belonging to other cultural backgrounds.

\section{THE TASKS OF EMOTIONAL DEVELOPMENT}

The Tasks of Emotional Development Test (Cohen \& Weil, 1971) [22] aims to assess the social and emotional adjustment of children from 6 to 18 years of age. The TED Test consists of four sets of 12 cards. Two sets consist of a parallel series for latency-age boys and girls. Various developmental tasks are portrayed on the cards, such as the establishment of trust in people, the mastery of aggressive feelings toward peers, the development of a conscience with respect to the property of others, and separation from the mother figure. The strengths of the test include elaborate scoring rules and standardized administration. The limitations are with regard to the out dated pictures and limited normative data available for only first 6 cards.

\section{THE ROBERTS APPERCEPTION TEST FOR CHILDREN}

The Roberts Apperception Test for Children (McArthur \& Roberts, 1982) [23] is for children from 6 to 15 years of age. The test aims to assess children's perceptions of common interpersonal situations, familial issues and adjustment. The cards have realistic black-and-white drawings of children interacting in everyday situations. Eleven of the 16 cards have parallel versions for girls and boys; the remaining 5 cards are not gender specific. A parallel version for black children is included. Each card refers to a certain developmental theme, such as aggression, fear, parent-child relationship, parental 
disagreement and sexuality. The process of administration is standardized and an elaborate scoring system is prescribed. The test has been standardized on 200 children. Discriminant Validity was established with studies on chronically ill children. The test also was found to have satisfactory inter rater agreement.

\section{THE FAMILY APPERCEPTION TEST}

The Family Apperception Test (Sotile, et al., 1988) [13] is based on family systems theory and is for children aged 6 years and older. The test aims to elicit projective responses pertaining to family process and structure and the effects associated with family relationships. There are 21 cards and depict life-like pencil drawings of children with family members in various scenes. The themes commonly elicited by each card include marital and family conflict, limit setting, boundary problems, dysfunctional circularity, open versus closed family systems, transgenerational alliances and conflicts, sexual abuse, and self-concept. There is a separate set of cards with depictions of black families, making it more culturally relatable to the black population. While the test is innovative, it lacks empirical evidence and a clear scoring technique.

\section{TELL ME A STORY}

Tell-Me-A-Story (Costantino, et al., 1988) [24] [25] is a culturally sensitive test for inner-city children( lesser priviledged) and adolescents of 5 to 18 year of age. The colour drawings of the TEMAS cards portray contemporary inner-city scenes with family and peer interactions. The test has 2 Forms, The Long Form entails administration of 23 cards and the short form consists of 9 cards. Eleven cards are sex-specific; the remaining cards are used with both sexes. The parallel minority version of the TEMAS shows Hispanic or black characters. The test aims at understanding of cognitive functions, personality functions and affective functions of the test taker. It is mainly used for getter a better understanding about a child's emotional and adjustment issues. The test administration is standardized and has a structured scoring technique. The test has been standardized on 643 children from 5- to 13-years aged children and adolescents. The test has a moderate internal consistency of .70. There is evidence of moderate concurrent and discriminant validity. The test is commendable for its contribution to multicultural assessment.

\section{THE CHILDREN'S APPERCEPTIVE STORY TELLING TEST (CAST)}

The Children's Apperceptive Story-Telling Test -CAST (Schneider, 1989) [13] was developed for children aged 6 to 13 years. The test is theoretically based on the Adlerian theory focusing on individual's social functioning. The test consists of color drawings on 17 cards that portray contemporary scenes with children from various ethnic groups in family, peer, and school situations. Fourteen cards have parallel male and female versions. The test has standardized administration process and systematic scoring techniques. The test has been standardized on 876 school going children. The authors report good internal consistency and inter rater reliability.

\section{THE CHILDREN'S SELF REPORT AND PROJECTIVE INVENTORY}

The Children's Self-Report and Projective Inventory (Ziffer \& Shapiro, 1992) [26] combines various assessment methods, including a set of thematic apperception cards. The 12 cards have simplified black-and-white drawings of characters whose faces have been left blank. Each card represents a specific theme, such as self-image, needs and fantasies, autonomy, initiative, family relationships, and peer relationships. The cards are to be administered in the standard fashion of a thematic apperception test. Since the probes are personalized, the responses are rather at a conscious level. Apart from this the test lacks standardized scoring, administration and normative data. 


\section{THE ADOLESCENT APPERCEPTION CARDS}

The Adolescent Apperception Cards (Silverton, 1993) [27] is designed specifically for adolescents (12 to 19 years). The response of the examinee is to create personal narratives based on cognitive schemas or mental structures with which the individual construes meaning for his experience of the world and that these narratives are an expression of the his conception of self and others. The test consists of 11 cards. Seven of the 11 cards are for both males and females; four cards have sex-specific parallel versions. Themes that may be elicited by each card, according to the manual, include loneliness, physical and sexual abuse, participation in unapproved activities (gang activity or drug abuse), sexual maturity, domestic violence, and parenting styles. The cards have realistic images and tap on important issues in adolescent years. However the scoring is highly subjective and it is not a standardized test.

The above section clearly highlights that use of pictures in projective testing has earned success, thereby leading to the development of the various tests reviewed. Projective Tests have received a lot a criticism due to issues in standardization, scoring and interpretation. However, evaluation of this is beyond the scope of this article. The review of use of pictures in projective testing clearly highlights how pictures are considered as relatable stimuli among children and adolescents. The relatable nature of pictures thereby enables the researcher/ clinician to elicit rich and experiential responses. The review also highlights the need and importance of clarity of pictures and using pictures relevant in today's world, else it makes the entire process of testing futile, as the test taker would spend more time understanding the picture. This would limit responses to a descriptive level and not manage to tap the experiences of the examinee. Another important highlight of this section would be the cultural relevance of pictures. While pictures are relatively culture fair, environmental details, clothing, ethnicity impacts the way in which children and adolescents perceives the picture, therefore, highlighting the importance of adaptations and parallel versions for different cultures and ethnicity as seen in the review.

\section{PICTURES IN RATING SCALES}

Use of pictures in rating scales is a rather new concept in psychological assessment, but is steadily picking up pace.

\section{PICTORIAL SCALE OF PERCEIVED COMPTENCE AND SOCIAL ACCEPTANCE FOR YOUNG CHILDREN}

The first test that used pictures in assessment was Pictorial Scale of Perceived Competence and Social Acceptance for Young Children [28]. The test was developed by Susan Harter and Pike in 1984 in the University of Denver. The test is mainly for children from 4 to 7 years of age. It aims to measure their cognitive and physical competence and social acceptance. The 24-item instrument is made up of four subscales comprising of six items each: Cognitive Competence, Physical Competence, Peer Acceptance, and Maternal Acceptance. There are separate versions were developed for two age groupings: Preschool to Kindergarten (Form PK), and First to Second Graders. The test typically takes 1015 minutes for administration. The test consists of two illustrations for each item which are polarised from which the child identifies with one (One picture with a child who is very good at the task and a second picture with a child who is not very good at the task). Each item is scored on a 4-point scale, where 4 represents the highest degree of perceived acceptance or competence. Higher scores reflect a greater sense of competence or social acceptance. The Pictorial Scales of Perceived Competence and Social Acceptance were devised as a downward extension of the Perceived Competence Scale for Children developed for children and adolescents 8 to 18 years old. The test has been standardized on 255 children who are preschoolers, kindergartners, first graders and second graders. The Reliability alpha coefficient for the sub scales was found to be between 0.53 and 0.83 . Studies that attempted to evaluate the validity of the test however correlations between teacher and self-ratings were found to be moderately weak. Despite its limitations, the test was clearly a breakthrough and pioneered the application of pictures in rating scales. 


\section{THE PRESCHOOL SYMPTOM SELF REPORT (PRESS)}

The Preschool Symptom Self-Report - PRESS (Martini et al., 1990) [29] is a measure to facilitate the self-report of depressive symptoms in preschool children, primarily focusing on symptoms accessible to the child's perception such as depressed mood, worrying, fatigue, fearfulness, and poor self-image. It is for children between 3 to 5 years of age. The time taken for administration is approximately 15 minutes. It consists of 25 items, each comprising two illustrations, similar to the Susan Harter scales. One illustration consists of the problem behaviour and the other illustrating the absence of that behaviour. The administrator reads an accompanying prompt with each illustration and the child is then asked to indicate "Which picture is more like you?" The test also includes a modified version that allows the parents and teachers to respond to the same pictures about the child. The test is translated in various languages too. The test has been standardized on a sample of 84 Head Start children and their parents and teachers. A parent-teacher version of the instrument correlated with other measures designed for adult rating of children's depression. The test was found to have a high alpha coefficient of 0.90 and an equally high intra class correlation of 0.86 .

\section{THINGS I HAVE SEEN AND HEARD}

Richters and Martinez developed the scale Things I Have Seen and Heard [30] in the year 1992. The scale aims to assess the frequency, through child self-report, of exposure to violence and violence related activities at home and in the community. It is a 20 item scale where the interviewer verbally asks a question related to the exposure of the child to violence. A pictorial format is used to facilitate child comprehension of response options. On the response form, five stacks of balls are depicted below each description of violence, each with a different number of balls, ranging from no balls (an empty circle) to four balls (representing many times). Children select 1 of 5 pictorial responses ranging from 0 (never) to 4 (many times). The test requires 5-10 minutes for administration. The initial pilot study was done on 152 first and second grade students from low-income, moderately violent neighborhoods in Southeast Washington, D.C. The test retest reliability was found to be high 0.81 .

\section{LEVONN - A CARTOON BASED INTERVIEW FOR ASSESSING CHILDREN'S DISTRESS SYMPTOMS}

Richters and Martinez further extended their work as part of a NIMH project in 1993 by developing Levonn: A Cartoon-Based Interview for Assessing Children's Distress Symptoms [31] over witnessing violence. The scale designed for children between the ages of 6 and 10 years. It consists of 42 items with cartoons to assess symptoms of depression, anxiety, intrusive thoughts, and sleep problems related to exposure to violence. Levonn assesses children's distress symptoms with that of a cartoon character named Levonn. Each item includes a 2 to 3 sentence script that the interviewer reads. Eg - Here is Levonn feeling very sad for a whole day. He gets up in the morning feeling sad, he feels sad all day and he still feels sad at bedtime. How many times have you felt like Levonn? Below each item, a visual analogue scale is presented with a picture of three thermometers, each with the mercury at one of three labelled levels: never, some of the time, and a lot of the time (The responses are scored from 1 to 3 . Higher the distress reported higher the score). Children are asked to report how often they have felt like Levonn by pointing to the thermometer that best represents the frequency with which they have experienced the same symptoms. Internal consistency reliability ranges from 0.71 to 0.84 and the test retest reliability score was found to be 0.81 .

\section{THE VIOLENCE EXPOSURE SCALE FOR CHILDREN - REVISED (VEX-R)}

The Violence Exposure Scale for Children, Revised (VEX-R) [32] developed by Fox \& Leavitt 1995 and revised in 2000. VEX $-\mathrm{R}$ is a 21 -item, cartoon-based interview intended to measure child self-reported exposure to violence. It is based on a measure designed by Richters and 
Martinez (1992) Things I've Seen and Heard. The scale measures the child's overall exposure to violence, both as a victim and as a witness. The violent incidents assessed are beating, chasing, robbery, threat with a weapon, shooting, stabbing, pushing or shoving, and slapping. The pictures show a central character, Chris, witnessing and experiencing violent events. The scale offers a cartoon depiction of each event in order to give the child a concrete reference point. Following the interviewer's explanation of what Chris is witnessing or experiencing in each picture, the children are asked how often they have been exposed to the same incident. Below each cartoon is a picture of four thermometers, each with the mercury at one of four labeled levels: never, once, a few times, and lots of times. Responses are measured on a 4-point Likert scale. There are separate forms available for boys and girls. Initial preliminary testing was done on $40 \mathrm{sub}$ urban children, mainly for the purpose of face validity of the items. As a result, several items were dropped due to a lack of clarity, and several new questions were incorporated. Internal consistency reliability of the VEX-R ranges from 0.80 to 0.86 .

\section{PICTORIAL INSTRUMENT FOR CHILDREN \& ADOLESCENTS (PICA-IIIR)}

Pictorial Instrument for Children and Adolescents -PICA III R (Ernst et.al.2000) [33] is a semi structured interview with 137 pictures organized in modules covering 5 diagnostic categories - anxiety disorders, mood disorders, psychotic disorders, disruptive behaviour disorders and substance abuse. The pictures are simple, schematic, gender neutral and refer to a single DSM -III-R criterion. The pictures encompass emotions, behaviour, thought content and thought processes. The gender is ambiguous, which was done with the rationale to facilitate identification of the child being interviewed. Each picture is accompanied by a set of comments and questions provided to help children understand the drawings and rate themselves. Once the picture is understood, the next questions probe the severity of the symptoms, frequency of occurrence and period of time when the symptom was present. The answer is rated on a five point visual analogue scale. A score of two or greater is considered positive. It can be used for children in from 6 to 16 years of age. It was validated on 51 inpatient children between ages 6 to 15 years. It provides a categorical and dimensional evaluation of childhood psychopathology. Internal Consistency Reliability was excellent for psychotic disorders (0.86), Anxiety (0.85), Depression (0.84), Oppositional Defiant Disorder/Conduct Disorder (0.84) and ADHD (0.80), moderate for Mania (0.69) and marginal for Obsessive Compulsive Disorder (0.54). Discriminant Validity was computed and the tool was found to discriminate significantly among diagnostic groups. Sensitivity of the instrument overtime was also checked by comparing severity scores at the time of hospitalization and before discharge. It found that the mean scores significantly declined.

\section{THE DOMINIC-R : A PICTORIAL INTERVIEW}

The Dominic-R: a Pictorial Interview [34] developed by Valla in 1997 and revised in 2000 is for children between 6 to 11years of age. It is a fully structured questionnaire based on the DSM IIIR to assess mental disorders in children. It is designed being sensitive to the cognitive immaturity of young children. The Dominic-R depicts a child named Dominic, either alone or with peers or adults, facing situations in the daily life of children. Instead of being organized in diagnostic modules, the pictures have been randomly mixed, with 8 normal situations intermixed with DSM-III-R abnormal behaviours. Drawings showing happy children in normal situations make the instrument more acceptable and less threatening for the test taker. The various disorders that the test measures are simple (specific) phobias, separation anxiety, overanxious (generalized anxiety), depression/dysthymia, attentiondeficit/hyperactivity, oppositional defiant, and conduct disorder. The test has various versions and formats. Terry is an alternate format for African-American children. Apart from this computerized versions are available for African-American, Asian, white, and Latino girls and boys. The test has translations in Spanish (Latino), German, and French versions. The time taken for administration is 15 to 25 minutes for paper version and 15 minutes for computerized versions. The responses for the questions are 'Yes' and 'No' answers are recorded on an appropriate scoring sheet. The test has been standardized on sample of 340 community children aged 6 to 11 years that was drawn from 4 schools located in a 
lower-middle class Montreal urban area. The test-retest values were 0.60 or more for 21 symptoms. As for disorder symptom scales, ICCs (Inter class correlations) between test and retest ranged from 0.71 to 0.81 . Criterion validity against clinical judgment yielded values ranging from 0.64 to 0.88 between Dominicbased diagnoses and DSM IIIR diagnoses based on each judge's clinical judgment. Cronbachs Alpha measuring internal consistency was found to be 0.89 for both internalizing (53 items) and externalizing (42 items) disorder scales.

\section{THE KOALA FEAR QUESTIONNAIRE (KFQ)}

The Koala Fear Questionnaire (KFQ) [35] is a standardized self-report scale for assessing fears and fearfulness developed by Muris et al., in 2003. It is a 31 items of potentially fearprovoking stimuli and situations that are all illustrated with pictures. Children rate the intensity of their fear of these stimuli by using a visual scale depicting Koala bears expressing various degrees of fear (no fear, some fear, a lot of fear). It is meant for children between the age ranges of 4 to 12 years. The psychometric properties of the test were established on 6 separate studies. In the first study, the test was administered on 108 primary children as a part of a pilot study and found that the visual fear scales of Koala bears as employed in the test are highly comparable to the standard 3-point scales that are used in other childhood fear measures. The findings of the test highly intercorrelated with Fear Survey Schedule for Children: FSSC-R (a standardized measure for assessment of fear among children) and yielded highly similar results. Thereby providing support for the concurrent validity of the visual fear scales of Koala bears. The second and third study on a sample of 165 primary school children, positive and significant associations emerged between the KFQ and all other measures such as FSSC-R, the trait anxiety version of the State-Trait Anxiety Inventory and the Spence Children's Anxiety Scale .Thereby establishing the convergent validity of the Koala Fear Questionnaire. Another study aimed at establishing reliability of the test on a sample of 189 primary school children. The test was found to have good internal consistency with an alpha of over 0.87 . The test-retest stability was found adequate with an ICC coefficient of 0.84 . The test was found to have good reliability and validity with younger age range children too. On a combined sample (of the previous studies) of over 900 children who completed the test was subjected to factor analysis. The factor structure of the KFQ was found to be theoretically meaningful: although the data clearly pointed in the direction of one factor of general fearfulness, spurs of the commonly found fivefactor solution of childhood fear measures were also found in the KFQ.

\section{PICTORIAL ANXIETY TEST (PAT)}

The most recently developed pictorial measure is The Pictorial Anxiety Test (PAT) [36] developed by Kathrin Dubi and Silvia Schneider in 2009. The test is for young children between 4 and 8 years of age. The aim of the test is to assess the tendency toward anxiety and avoidance in specific situations using hypothetical vignettes. The test has 17 situations and provides three main scores (total anxiety score, total avoidance score, composite score). PAT assesses a wide range of anxieties and fears on a dimensional level, omitting excessive focus from duration and onset which children find difficult to report. The test is supplemented by a structured interview conducted with the parents of the children. Each item of the PAT includes two color illustrations, depicting two different responses of a child in a potentially fearful situation similar to the Susan Harter scales. The child's degree of fear and avoidance is assessed on a 4-point-scale ranging from "not at all" (0) to "very much" (3). The test has separate forms for boys and girls. The test was standardized on a sample of 71 children between 5 and 7years of age who were enrolled in a Swiss study of the behavioral, cognitive, and psycho physiological features and treatment of children with separation anxiety. Cronbach's Alphas for PAT was 0.87 for the composite score, 0.76 for the anxiety score and 0.77 for the avoidance score, indicating adequate to good internal reliability. Test-retest reliability was found to be acceptable in children's composite scores, anxiety scores, and avoidance scores on the PAT over a 4-6 weeks period. The convergent validity of the PAT scores was supported by moderate correlations between the PAT scores and a widely used measure of childhood anxiety, namely the Revised Children's Manifest Anxiety Scale (RCMAS). 
The above section reviewed pictorial tools that are based on self report format. Application of pictures in self report format is an innovative technique that has been there for a little over three decades now. However, it has been steadily making a niche of its own in the area of assessment with children and adolescents. Most of the tests available and reviewed qualify as a reliable and valid test with sound psychometric properties. Going beyond the technical details, the use of pictures in self report measures of psychological assessment with children and adolescents have four very strong advantages that are noteworthy.

1. Firstly, use of pictures increases comprehensibility of items. Difficulty in comprehension of items and guess based responses are often pitfalls in self report format. Therefore use of pictures makes the responses devoid of chance factors and can be considered as a reliable feedback of the test taker.

2. Secondly, the primary advantage of self report format is the objectivity that it brings to testing. However application of self report format with children below 10 years of age has been a challenge. Thereby highlighting a void for an objective form of assessment with younger children. Use of pictures in self report format successfully fills in this void. Thereby making pictorial tools with younger children particularly useful.

3. Self Report Formats are often considered as dry and is criticized for not being able to capture a holistic viewpoint that is important in psychological assessment. Use of pictures in assessment makes the process less threatening. It provides the clinician with information of subtle nuances about the child's behavior while viewing a picture and the details of the picture which the child chooses to acknowledge or miss. This gives richer information and taps on finer details in assessment.

4. Usefulness of use of picture based assessment in violence prone areas and in emotional disorders is evident from the review.

Therefore use of pictures in self report format with children and adolescents is both objective and comprehensive. The scope and application of this format is very promising. However it must be noted that it useful in adjunct with other techniques of assessment and multiple informants as no form of assessment is precise in isolation.

\section{SUMMARY AND CONCLUSIONS}

Use of pictures in psychological assessment with children and adolescents has come a long way. There has been considerable movement from being a subtest in intelligence to a primary format in psychological assessment in the domain of cognitive, projective as well as self report format of testing. Pictorial formats have made a niche and appears to have a lot of scope due to the innumerous advantages that they come with like the culture fair nature of the stimulus, the fact that it facilitates better comprehension, the richer information that it is able to elicit, the ease it brings to testing, lesser threatening nature of the material, the objectivity it retains, reduces chance/guess responses and most importantly it's applicability with younger children and children from special populations.

The challenges include difficulty in standardizations as pictorial testing requires individual administration making the process of standardization labour intensive and time consuming. Another challenge that the review highlights is adaptations that pictorial tools require to cultural context and ethnicities, as in order to facilitate identification the children will need to relate to the picture thereby highlighting the attention that needs to be given to background details in pictures, culturally sensitive information and ethnicity. However it must be noted here that mere downward extension of tests from adult to child samples is not enough. In fact there is clearly scope to develop children's pictorial scales or pictorial versions of scales to understand constructs like emotions, relations, attachments and experiences to name a few.

The review also brings to light the lack of pictorial tools developed in the Indian context. In fact the only available Pictorial tools in the Indian context are Indian adaptations of Peabody Picture Vocabulary Test, Thematic Apperception Test, Children's Apperception Test and Picture 
Frustration Test, therefore, highlighting both the need and scope for development of pictorial scales for children and adolescents in India.

It would be apt to conclude the review with a quote highlighting the value of images or pictures.

"Images seem to speak to the eye, but they are really addressed to the mind. They are ways of thinking, in the guise of ways of seeing. The eye can sometimes be satisfied with form alone, but the mind can be satisfied with meaning, which can be contemplated, more consciously or less, after the eye is closed."

Duff (1975), a media theorist [37]

\section{REFERENCES}

1. Freeman M, Mathison S. Researching Children's Experiences. (1st ed.). New York: The Guilford Press ; 2009.

2. Schratz M, Walker R. Research as social change: New opportunities for qualitative research. New York: Routledge ; 1995.

3. Greene S, Hogan D. Researching Children's Experiences : Methods and Approaches. (1st, 2nd reprint ed.). : Sage Pubications ; 2005.

4. Vygotsky L. Mind in Society: The development of higher psychological processes. Cited In Greene S, Hogan D. Researching Children's Experiences : Methods and Approaches. (1st, 2nd reprint ed.). : Sage Pubications ; 2005.

5. Anastasi A, Urbina S. Psychological Testing. (7th ed.). New Jersey, USA: Prentice Hall ; 1997.

6. Community - University Partnership for the study of Children, Youth, and Families. Review of the Peabody Picture Vocabulary Test, Fourth Edition [PPVT -4]. Edmonton, Alberta, Canada ; 2011.

7. Kush JC. Review of the Peabody Picture Vocabulary Test, Fourth Edition. Mental Measurements Yearbook, $18 ; 2008$.

8. Dunn L. (2007). Pearson. Retrieved 21February,2016, from http://www.pearsonclinical.com/language/products/100000501/peabody-picture-vocabulary-test-fourth-

edition-ppvt-4.html

9. Verma SK. Index of cognitive development. In Channabasavanna SM, Varghese M, Chandra PS. Indicators of mental Health - A multi centre ICMR study: Report of the study and manual for family interventions by the health worker. New Delhi: ICMR ; 1995.

10. Murray HA. Thematic Apperception Test: Manual. Cambridge, MA: Harvard University Press ; 1943.

11. Rapaport D. Principles underlying projective techniques. In M.M. Gill (Ed.), Collected papers of David Rapaport (pp. 91-97). New York: Basic Books ; 1967.

12. Choudhary U. Indian Adaptation of TAT. New Delhi: Manasayan ; 1960.

13. Kroon N, Goudena P, Rispens J Thematic apperception tests for child and adolescent assessment: A practitioner's consumer guide. J Psychol Assess 1998;16:99-117.

14. Blum GS. The Blacky Pictures: A technique for the exploration of personality dynamics. New York: Psychological Corporation ; 1950.

15. Rosenzweig S. The Rosenzweig Picture-Frustration Study, Children's Form. In Zubin J, Freedman A.(Eds), Projective techniques with children (pp. 149-176). New York: Grune \& Stratton ; 1960.

16. Pareek U, Rosenzweig S. Manual of The Indian Adaptation of Rosenzweig Picture Frustration Study (Children's Form). New Delhi: Manasayan ; 1989.

17. Bellak L, Bellak SS. Children's Apperception Test: Manual (8th rev. ed.). Larchmont, NY: CPS ; 1991.

18. Choudhary U. Indian Adaptation of CAT. New Delhi: Manasayan ; 1985.

19. Lee SG. Review of the Four Pictures Test. In O.K. Buros (Ed.), The sixth mental measurements yearbook (pp. 1237-1239). Highland Park, NJ: Gryphon Press ; 1965.

20. Rabin AI. (Ed). Projective Techniques in Personality Assessment. New York: Springer Publications ; 1968.

21. Langeveld MJ. The Columbus: Picture analysis of growth towards maturity. Basel, Switzerland: Krager ; 1969.

22. Cohen H, Weil GR. Tasks of Emotional Development: A projective test for children and adolescents. Brookline, MA: Massachusetts School of Professional Psychology ; 1975. 
23. McArthur DS, Roberts GE. Roberts Apperception Test for Children: Manual. Los Angeles: Western Psychological Services ; 1982.

24. Constantino G, Malgady R. Development of TEMAS, A multicultural thematic apperception test: Psychometric properties and clinical utility. In Sodowsky GR, Impara J. (Eds.). Multicultural assessment in counseling and clinical psychology (pp. 85-136). Lincoln, NE: Buros Institute of Mental Measurements ; 1996.

25. Ritzler B. TEMAS (Tell-Me-A-Story): Review. J Psychoeduc Assess 1993;11:381-9.

26. Ziffer RL, Shapiro LE. Children's self-report and projective inventory. Narberth, PA: Psychological Assessment Services ; 1992.

27. Silverton L. Adolescent Apperception Cards: Manual. Los Angeles: Western Psychological Services ; 1993.

28. Harter S, Pike R. The pictorial scale of perceived competence and social acceptance for young children. Child Dev 1984;55:1969-82.

29. Martini DR, Strayhorn JM, Puig AJ. A symptom self-report measure for preschool children. J Am Acad Child Adolesc Psychaitry 1990;29(4):594-600.

30. Richters JE, Martinez P. Things I Have Seen and Heard: A Structured Interview for Assessing Young Children's Violence Exposure. Rockville MD, National Institute of Mental Health ; 1992.

31. Martinez P, Richters JE. The NIMH community violence project: II. Children's distress symptoms associated with violence exposure. Psychiatry 1993;56:22-35.

32. Shahinfar A, Fox NA, Leavitt LA. Preschool Children's Exposure to Violence : Relation of Behavior Problems to Parent and Child Reports. Am J Orthopsychiatry 2000;70(1):115-25.

33. Ernst M, Cookus BA, Moravec BC. Pictorial instrument for children and adolescents (PICA-III-R). J Am Acad Child Adolesc Psychiatry 2000;39:94-9.

34. Valla JP, Bergeron L, Smolla N. The Dominic-R: A pictorial interview for 6- to 11-year-old children. J Am Acad Child Adolesc Psychiatry 2000;39:85-93.

35. Muris P, Meesters C, Mayer B, Bogie N, Luijten M, Geebelen E, et al. The Koala Fear Questionnaire: A standardized self-report scale for assessing fears and fearfulness in pre-school and primary school children. Behav Res Ther 2003;41:597-617.

36. Dubi K, Schneider S. The Picture Anxiety Test [PAT]: A new pictorial assessment of anxiety symptoms in young children. J Anxiety Disord 2009;23:1148-57.

37. Duff W. Images, Stone, BC: Thirty centuries of northwest coast Indian sculpture. Saanichton, British Columbia: Hancock House ; 1975.

$$
\begin{aligned}
& \text { Acknowledgements - Nil } \\
& \text { Source of Funding - Nil } \\
& \text { Conflict of Interest - Nil }
\end{aligned}
$$

